\title{
Resilience of Alzheimer's Disease to COVID-19
}

Jingwen $\mathrm{Li}^{\mathrm{a}, 1}$, Xi Long ${ }^{\mathrm{b}, 1}$, Heqing Huang ${ }^{\mathrm{c}, 1}$, Jine Tang ${ }^{\mathrm{d}, 1}$, Chunli Zhu ${ }^{\mathrm{e}}$, Shaoping $\mathrm{Hu}^{\mathrm{f}}$, Jing $\mathrm{Wu}^{\mathrm{g}}$, Jinghong $\mathrm{Li}^{\mathrm{h}, *}$, Zhicheng Lin ${ }^{\mathrm{i}, *}$ and Nian Xiong ${ }^{\mathrm{a}, \mathrm{e}, *}$

${ }^{a}$ Department of Neurology, Union Hospital, Tongji Medical College, Huazhong University of Science and Technology, Wuhan, Hubei, China

${ }^{\mathrm{b}}$ Department of Radiology, Union Hospital, Tongji Medical College, Huazhong University of Science and Technology, Wuhan, Hubei, China

${ }^{\mathrm{c}}$ Department of Pediatrics, People's Hospital of Dongxihu District, Wuhan, Hubei, China

${ }^{\mathrm{d}}$ Department of Science and Education, Wuhan Red Cross Hospital, Wuhan, Hubei, China

${ }^{\mathrm{e}}$ Department of Neurology, Wuhan Red Cross Hospital, Wuhan, Hubei, China

${ }^{\mathrm{f}}$ Department of Radiology, Wuhan Red Cross Hospital, Wuhan, Hubei, China

${ }^{\mathrm{g}}$ Department of Epidemiology and Biostatistics, School of Public Health, Tongji Medical College, Huazhong University of Science and Technology, Wuhan, Hubei, China

${ }^{\mathrm{h}}$ Department of Medicine, University of California San Diego, La Jolla, CA, USA

${ }^{\mathrm{i}}$ Laboratory of Psychiatric Neurogenomics, McLean Hospital, Harvard Medical School, Belmont, MA, USA

Accepted 1 August 2020

\begin{abstract}
.
Background: Facing the novel coronavirus disease 2019 (COVID-19), most vulnerable individuals are seniors, especially those with comorbidities. More attention needs to been paid to the COVID-19 patients with Alzheimer's disease (AD), which is the top age-related neurodegenerative disease.

Objective: Since it is unclear whether AD patients are prone to COVID-19 infection and progression to severe stages, we report for the first time a retrospective analysis of the clinical characteristics of AD patients with COVID-19 pneumonia.

Methods: We conducted a retrospective cohort study of the clinical data of 19 AD patients with COVID-19 pneumonia, compared with 23 non-AD COVID-19 patients admitted at the same time to our hospital. Demographic, clinical, laboratory, radiological, and treatment data were collected and analyzed.

Results: Between AD patients and non-AD patients with COVID-19 pneumonia, the pneumonia severity was not significantly different. AD patients had a higher clustering onset than non-AD patients. The median duration from symptom onset to hospitalization were shorter in AD patients than non-AD patients, indicating the former were sent to the hospital by their family or from nursing home earlier than the later. The median duration from hospitalization to discharge seemed shorter in $\mathrm{AD}$ patients than non-AD patients. Dementia patients seemed less likely to report fatigue. It is noticed that more AD patients might have pericardial effusion than the non-AD patients.

Conclusion: AD patients with COVID-19 were in milder conditions with a better prognosis than non-AD patients. AD patients who had adequate access to healthcare showed resilience to COVID-19 with shorter hospital stays.
\end{abstract}

Keywords: Alzheimer's disease, clinical characteristics, COVID-19

\footnotetext{
${ }^{1}$ These authors contributed equally to this work.

*Correspondence to: Jinghong Li, Department of Medicine, University of California San Diego, La Jolla, CA 92093, USA. E-mail: ji1055@health.ucsd.edu; Zhicheng Lin, Laboratory of Psychiatric Neurogenomics, McLean Hospital, Harvard Medical School, Belmont, MA 02478, USA. E-mail:
}

zhicheng_Lin@hms.harvard.edu; Nian Xiong, Department of Neurology, Union Hospital, Tongji Medical College, Huazhong University of Science and Technology, 1227 Jiefang Ave, Wuhan, Hubei, China; President of Wuhan Red Cross Hospital, 392 Hongkong Road, Wuhan, Hubei, China. E-mail: nianxiong@hust.edu.cn. 


\section{INTRODUCTION}

Facing the novel coronavirus disease 2019 (COVID-19), most vulnerable individuals are those with comorbidities especially in elderly population. About $15 \%$ of confirmed cases progress to the severe COVID-19 pneumonia with Acute Respiratory Distress Syndrome (ARDS). The highest fatality rate of COVID-19 pneumonia was reported for older adults and individuals with weakened immune systems [1]. Since Alzheimer's disease (AD) is a well-known degenerative disease usually affecting the elderly population [2], it is unclear whether $\mathrm{AD}$ patients are prone to be infected with COVID-19 and develop to severe stages. In general, mortality from pneumonia has been reported to be twice as high in individuals with dementia compared to those without dementia [3]. Previous studies on COVID-19 mainly focused on epidemiological, clinical, and radiological features of the patients with confirmed infection [4, 5]. Here we report for the first time a retrospective analysis of the clinical data of $19 \mathrm{AD}$ patients (diagnosed according to the 2011 National Institute on Aging (NIA) - Alzheimer's Association (AA) diagnostic guidelines [6]) with COVID-19 pneumonia, compared with non-AD patients in our hospital.

\section{METHODS}

\section{Participants}

This is a retrospective cohort study of two groups, 19 consecutive AD patients and 23 age-matched non-AD patients with novel coronavirus pneumonia admitted from January 23, 2020 to March 19, 2020 at Wuhan Red Cross Hospital (WRCH) in Wuhan, China. 19 patients had a clinical diagnosis of probable AD according to NIA-AA diagnostic guidelines. Inclusion criteria included a Mini-Mental State Examination (MMSE) score $>19$, age between 65-100 years, and willingness to participate in the study. Exclusion criteria were other neurological diseases causing cognitive decline (including severe cerebrovascular disease judged from cranial computed tomography or magnetic resonance imaging) and severe psychiatric diseases such as schizophrenia and major depression. All patients were diagnosed with COVID-19 according to the World Health Organization interim guidance, with positive reversetranscription polymerase chain reaction (RT-PCR) for SARS-CoV-2 (Supplementary Material 1).

\section{Data collection}

Epidemiological, demographic, clinical, laboratory, computed tomography (CT) imaging features, and treatment data were collected and analyzed. All data were checked for accuracy by two trained physicians (J.L. and N.X.). Patients were asked about basic information, medical history, exposure history, symptoms, and comorbidities. As a part of standard care, complete blood cell count, tests of kidney and liver function, and measurement of C-reactive protein and lactate dehydrogenase levels were performed. All patients received supportive therapy, including supplemental oxygen when saturations dropped below $92 \%$ as measured by pulse oximeter. Patients clinically suspected of having community-acquired pneumonia were administered with antibiotics empirically. Additionally, we use CT scores to evaluate the degree of involvement for five lung lobes. An overall lung "total severity score" was reached by summing the five lobe scores (range of possible scores, 0-25) (Supplementary Material 2) [7]. The study was approved by the WRCH Ethics Committee of the Wuhan Red Cross Hospital and written informed consent obtained from each subject before enrolment, followed by retrospective data collection.

\section{Statistical analysis}

Categorical variables were described as frequency rates and percentages, and continuous variables, using a median, minimum or maximum grade. SPSS (Statistical Package for the Social Sciences, version 25) was used for all statistical analyses, with $p<0.05$ considered as statistically significant. Continuous variables were expressed as median (range) and compared between independent groups by twotailed Student's $t$ tests; categorical variables were expressed as number (\%) and compared by $\chi^{2}$ tests or Fisher's exact tests; Bonferroni correction was used for multiple testing.

\section{RESULTS}

All patients had positive RT-PCR test results for SARS-CoV-2. There was no statistically significant difference in age between the two COVID-19 groups, $A D$ versus non- $A D$, although the females in the $A D$ group were on average older than those in the non$\mathrm{AD}$ group. In the $\mathrm{AD}$ group, $68.4 \%$ were females and the age range was 69 to 100 years of age. The AD patients had a higher clustering onset than the non- 
Table 1

Baseline characteristics and laboratory results of $19 \mathrm{AD}$ patients and 23 non-AD patients with COVID-19

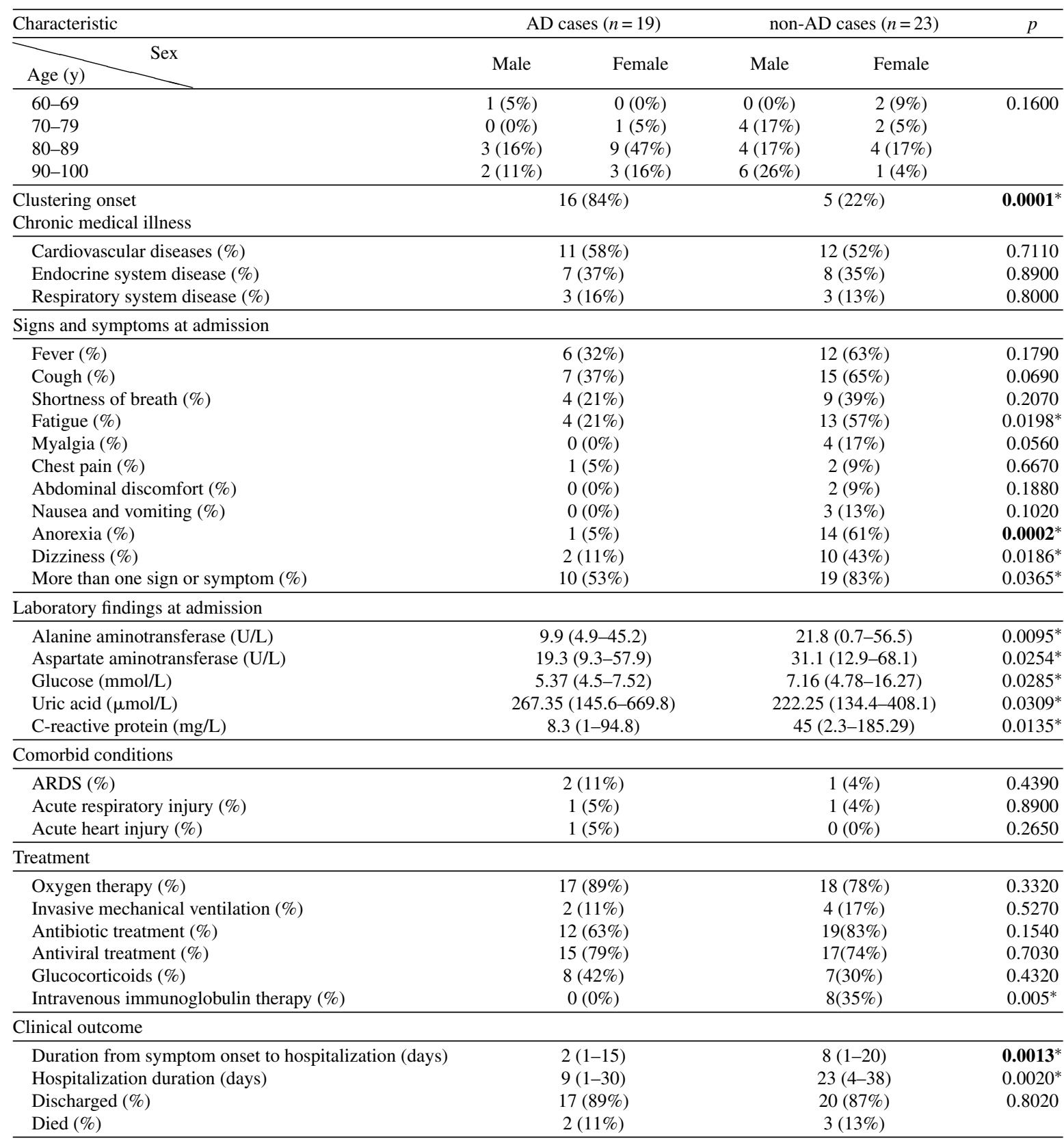

AD, Alzheimer's disease; ARDS, Acute Respiratory Distress Syndrome. Data are median, (range) or N (\%). *statistic difference by nominal $p<0.05$, comparing AD patients and non-AD patients were from $\chi^{2}$ tests or Fisher's exact tests. Bold, statistically significant after Bonferroni correction.

AD patients $(84 \%$ versus $22 \% ; p<0.0001)$. Besides dementia, some of them had comorbidities such as cardiovascular diseases (58\%) and endocrine system disease $(37 \%)$. The median duration from onset of symptoms to hospitalization was much shorter in the AD patients than the non-AD patients $(2$ versus 8 days; $p=0.0013$ ). Among the $19 \mathrm{AD}$ patients, there were fever, cough, and shortness of breath, similar to those in the non-AD patients $[4,5]$. Fatigue was less likely to be a complaint by the AD patients ( $21 \%$ versus $57 \% ; p=0.0198$ ), probably because these patients reported this symptom in a delayed manner. Only one 

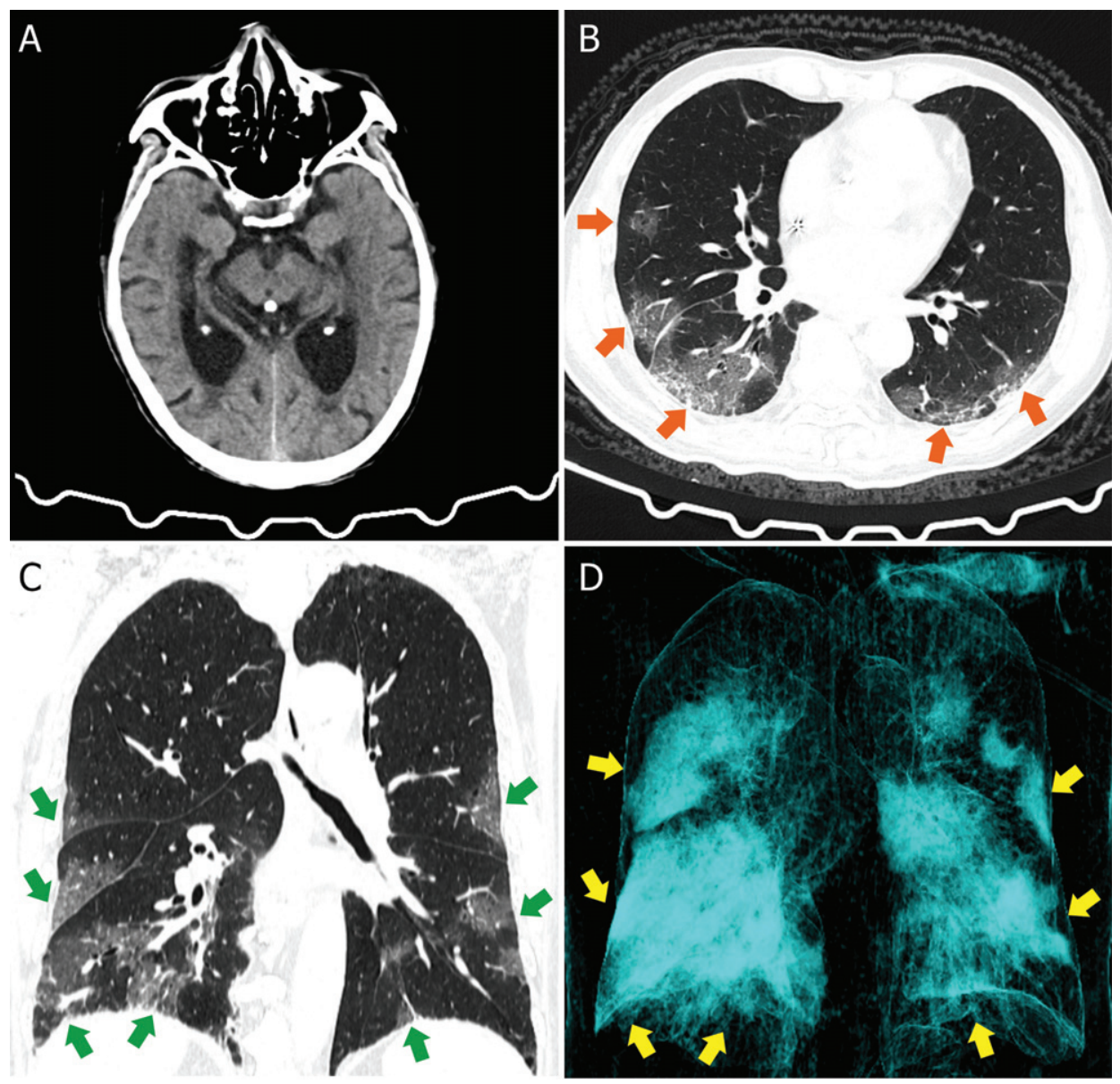

Fig. 1. Head and Chest CT images of an AD patient. A) Head CT image showed cerebral atrophy. B) Axis scan chest CT image showed bilateral patchy ground-glass opacities (orange arrows). C) Corona scan chest CT image showed bilateral multifocal ground-glass opacities (green arrows). D) Three-dimensional reconstruction image showed the lesions (yellow arrows).

AD patient $(5 \%)$ had anorexia, compared to more than a half of non-AD patients $(61 \%)$ experiencing this symptom $(p=0.0002)$. AD patients had less dizziness as well $(p=0.0186)$.

The time from hospitalization to discharge of the AD patients ranged from 1 to 30 days, with a median of 9 days, which was much shorter than the duration for the non-AD group whose median was 23 days (range, 4-38; $p=0.0020$ ). Similar treatments (i.e., oxygen therapy, non-invasive and invasive mechanical ventilation, antiviral and antibiotics treatment) were provided for all patients in both groups. None of the AD patients were treated with intravenous immunoglobulin therapy, compared with $35 \%$ nonAD patients with this treatment $(p=0.0050)$. There was no significant difference in the outcome of survival and death between these two groups.
COVID-19 related index levels like ALT, AST, and glucose were on average all lower in the AD group than the non-AD group (ALT, $p=0.0095$; AST, $p=0.0254$; glucose, $p=0.0285$ ). The median CRP level was lower in the $\mathrm{AD}$ patients than the non-AD patients ( 8.3 versus $45 ; p=0.0135$ ). Considering the fact that increased levels of these indexes were typical of COVID-19 [4, 8, 9], these laboratory results suggested that the acute inflammatory response seemed weaker in the AD than the non-AD patients. Additionally, uric acid levels were significantly increased in AD patients $(p=0.0309)$ (Table 1).

Chest CT scans revealed consistent differences between the two groups. Inevitably, both groups showed ground-glass opacification, which was characteristic of COVID-19, as shown by CT images of a representative $\mathrm{AD}$ patient in Fig. 1. Fewer AD 
Table 2

Imaging findings of two groups with COVID-19

\begin{tabular}{|c|c|c|c|}
\hline $\mathrm{NO}(\%)$ & $\begin{array}{c}\text { AD cases } \\
(n=19)\end{array}$ & $\begin{array}{c}\text { non-AD cases } \\
(n=23)\end{array}$ & $p$ \\
\hline \multicolumn{4}{|l|}{ Distribution } \\
\hline Periphery distribution & $2(11 \%)$ & $5(22 \%)$ & \multirow[t]{2}{*}{0.3320} \\
\hline Bilateral involvement & $17(89 \%)$ & $18(78 \%)$ & \\
\hline \multicolumn{4}{|l|}{ Number of lobes involved } \\
\hline 1 & $0(0 \%)$ & $1(4 \%)$ & \multirow[t]{5}{*}{0.5970} \\
\hline 2 & $4(21 \%)$ & $4(17 \%)$ & \\
\hline 3 & $0(0 \%)$ & $1(4 \%)$ & \\
\hline 4 & $6(32 \%)$ & $4(17 \%)$ & \\
\hline 5 & $9(47 \%)$ & $13(57 \%)$ & \\
\hline \multicolumn{4}{|l|}{ Lobe of lesion distribution } \\
\hline Left upper lobe & $15(79 \%)$ & $16(70 \%)$ & \multirow[t]{5}{*}{0.9360} \\
\hline Left lower lobe & $19(100 \%)$ & $19(83 \%)$ & \\
\hline Right upper lobe & $15(79 \%)$ & $20(87 \%)$ & \\
\hline Right middle lobe & $11(58 \%)$ & $16(70 \%)$ & \\
\hline Right lower lobe & $17(89 \%)$ & $22(96 \%)$ & \\
\hline \multicolumn{4}{|l|}{ Patterns of the lesion } \\
\hline Ground glass opacification & $19(100 \%)$ & $19(83 \%)$ & 0.0560 \\
\hline Consolidation & $6(32 \%)$ & $13(57 \%)$ & 0.1060 \\
\hline Mixed ground glass opacity and consolidation & $4(21 \%)$ & $15(65 \%)$ & $0.0004^{*}$ \\
\hline Crazy paving pattern & $2(11 \%)$ & $10(43 \%)$ & $0.0186^{*}$ \\
\hline Linear opacities combined & $11(58 \%)$ & $18(78 \%)$ & 0.1550 \\
\hline Interlobular septal thickening & $13(68 \%)$ & $19(83 \%)$ & 0.2830 \\
\hline Air bronchogram sign & $1(5 \%)$ & $4(17 \%)$ & 0.2270 \\
\hline \multicolumn{4}{|l|}{ Other findings } \\
\hline Adjacent pleura thickening & $14(74 \%)$ & $22(96 \%)$ & 0.0429 \\
\hline Bronchitis & $12(63 \%)$ & $14(61 \%)$ & 0.8790 \\
\hline Pulmonary emphysema & $8(42 \%)$ & $10(43 \%)$ & 0.9290 \\
\hline Pleural effusion & $8(42 \%)$ & $11(48 \%)$ & 0.7110 \\
\hline Pericardial effusion & $6(32 \%)$ & $1(4 \%)$ & $0.0184^{*}$ \\
\hline Lymphadenopathy & $8(42 \%)$ & $12(52 \%)$ & 0.5160 \\
\hline Total CT Score (median, range) & $5(2-20)$ & $9(1-25)$ & $0.0280^{*}$ \\
\hline
\end{tabular}

Data are $\mathrm{N}(\%)$ or median (range). ${ }^{*}$ statistic difference by nominal $p<0.05$, comparing AD patients and non-AD patients were from $\chi^{2}$ tests or Fisher's exact tests. Bold, statistically significant after Bonferroni correction.

patients $(21 \%)$ showed mixed pattern of ground-glass opacity and consolidation, compared with $65 \%$ of the non-AD patients with this image feature, but there is no statistic difference. Crazy paving pattern was shown in only 2 AD patients, compared with 10 non-AD patients $(p=0.0186)$. It is noticed that more AD patients had pericardial effusion than the non-AD patients $(32 \%$ versus $4 \% ; p=0.0184)$ (Table 2). All these compared indexes indicated that the AD patients had relatively mild conditions due to the timely treatment. In other words, the AD group received early treatment in the hospital so that their symptoms were milder than the non-AD group.

\section{DISCUSSION}

We present for the first time a retrospective cohort study of clinical features of a group of AD COVID-
19 pneumonia patients and age-matched non-AD COVID-19 pneumonia patients. Our results demonstrated that $\mathrm{AD}$ and non-AD patients had similar clinical presentations of COVID-19 pneumonia. AD patients have remarkably good appetites, which may interpret the less reported anorexia in our study. They received similar treatment after hospitalization. Although AD patients were sent to the hospital earlier from onset of symptoms, there was no significant difference in the outcome of survival and death between these two groups. However, AD patients had a conspicuously shorter median duration of hospital stay. The majority of our $\mathrm{AD}$ patients lived in nursing homes so they had adequate and prioritized access to healthcare, leading to earlier diagnosis and treatment for shorter hospital stays $[10,11]$.

Dementia-related characteristics may increase the risks of COVID-19 pneumonia. For example, people with $\mathrm{AD}$ may forget to wash hands or maintain social 
distancing, in addition to taking other precautions to prevent COVID-19 [12]. In addition, a previous report mentioned that older patients with dementia and COVID-19 infection may present with mild and atypical symptoms, namely diarrhea or drowsiness [11]. With timely access to healthcare, the outcome of AD patients with COVID-19 was not significantly different from that of non-AD patients. On the other hand, AD patients achieved a similar recovery rate with significant shorter hospital stays, compared to the non-AD patients.

More interestingly, we hypothesize that angiotensin-converting enzyme 2 (ACE2) is another likely mechanism for resilience of $\mathrm{AD}$ patients to COVID-19. Previous research has shown that the SARS-CoV-2 uses its spike protein S1 to enter cells by interacting with ACE2 receptor on cell surface membrane [13]. Consistently, it has been reported that ACE2 expression is reduced in AD patients [14]. Animal models have confirmed the reduced ACE2 expression in AD [15, 16]. Currently, investigations are ongoing to study activation of ACE2 versus cognition decline [17]. Based on our results of a retrospective cohort study, we postulate that the shorter hospitalization may be related to reduced expression of ACE2 receptor in $\mathrm{AD}$ COVID-19 patients.

Similar outcomes between AD and non-AD group patients may be due to timely treatment, professional caring of nursing home, and the ACE2 factor mentioned above. We are also interested in whether the E4 variant of apolipoprotein E (APOE4), the main susceptibility gene for $\mathrm{AD}$ [18], is involved in the protective effect and the better prognosis of our AD group patients with COVID-19. Presence of APOE4 allele has always been recognized as a risk factor for $\mathrm{AD}$. But a recent study reported the AD patients who carried APOE4 had less chronic diseases [19]. We are going to conduct a related exploration in the near future.

This study provides an early investigation of $\mathrm{AD}$ patients with COVID-19 pneumonia. But there were also some limitations in this study. Firstly, the sample size of our study was small. Secondly, our AD group mostly came from nursing homes, with higher exposure to infection but better care, which may explain the shorter hospital duration on average and the better conditions. Longitudinal studies with larger cohorts at different social settings would help to better understand how to manage vulnerable AD patients, especially those in nursing homes, with COVID-19 pneumonia.

\section{ACKNOWLEDGMENTS}

This work was supported by grants 2016YFC1306600 and 2018YFC1314700 from the National Key R\&D Program of China, grants 81873782 from the National Natural Science Foundation of China, Grant 2016CFB624 from Natural Science Foundation of Hubei Province, Grant 2017050304010278 from The Youth Science and technology morning light program of Wuhan City, 2018 Hubei medical research project WJ2019F030, 2018 Wuhan medical research project S201802140011, 2018 Wuhan Young and Middle-aged medical Talents Program and 2017 Hubei provincial Party Committee Organization Department the second batch of Hubei youth elite development plan (all to NX). The funding sources plays no role in the study design, data collection and manuscript writing.

Authors' disclosures available online (https:// www.j-alz.com/manuscript-disclosures/20-0649r1).

\section{Available of data and material}

All data included in this study are available upon request by contact with the corresponding author. The lead authors and manuscript's guarantor affirm that the manuscript is an honest, accurate, and transparent account of the study being reported; that no important aspects of the study have been omitted; and that any discrepancies from the study as planned have been explained.

\section{SUPPLEMENTARY MATERIAL}

The supplementary material is available in the electronic version of this article: https://dx. doi.org/10.3233/JAD-200649.

\section{REFERENCES}

[1] Wu Z, McGoogan JM (2020) Characteristics of and important lessons from the coronavirus disease 2019 (COVID-19) outbreak in China: Summary of a report of 72,314 cases from the Chinese Center for Disease Control and Prevention. JAMA. doi: 10.1001/jama.2020.2648

[2] Raber J, Huang Y, Ashford JW (2004) ApoE genotype accounts for the vast majority of AD risk and AD pathology. Neurobiol Aging 25, 641-650.

[3] Brown EE, Kumar S, Rajji TK, Pollock BG, Mulsant $\mathrm{BH}$ (2020) Anticipating and mitigating the impact of the COVID-19 pandemic on Alzheimer's disease and related dementias. Am J Geriatr Psychiatry 28, 712-721. 
[4] Huang C, Wang Y, Li X, Ren L, Zhao J, Hu Y, Zhang L, Fan G, Xu J, Gu X, Cheng Z, Yu T, Xia J, Wei Y, Wu W, Xie X, Yin W, Li H, Liu M, Xiao Y, Gao H, Guo L, Xie J, Wang G, Jiang R, Gao Z, Jin Q, Wang J, Cao B (2020) Clinical features of patients infected with 2019 novel coronavirus in Wuhan, China. Lancet 395, 497-506.

[5] Chen N, Zhou M, Dong X, Qu J, Gong F, Han Y, Qiu Y, Wang J, Liu Y, Wei Y, Xia J, Yu T, Zhang X, Zhang L (2020) Epidemiological and clinical characteristics of 99 cases of 2019 novel coronavirus pneumonia in Wuhan, China: A descriptive study. Lancet 395, 507-513.

[6] McKhann GM, Knopman DS, Chertkow H, Hyman BT, Jack CJ, Kawas CH, Klunk WE, Koroshetz WJ, Manly JJ, Mayeux R, Mohs RC, Morris JC, Rossor MN, Scheltens P, Carrillo MC, Thies B, Weintraub S, Phelps $\mathrm{CH}$ (2011) The diagnosis of dementia due to Alzheimer's disease: Recommendations from the National Institute on Aging-Alzheimer's Association workgroups on diagnostic guidelines for Alzheimer's disease. Alzheimers Dement 7, 263-269.

[7] Pan F, Ye T, Sun P, Gui S, Liang B, Li L, Zheng D, Wang J, Hesketh RL, Yang L, Zheng C (2020) Time course of lung changes on chest CT during recovery from 2019 novel coronavirus (COVID-19) pneumonia. Radiology 295, 715721.

[8] Wang D, Hu B, Hu C, Zhu F, Liu X, Zhang J, Wang B, Xiang H, Cheng Z, Xiong Y, Zhao Y, Li Y, Wang X and Peng Z (2020) Clinical characteristics of 138 hospitalized patients with 2019 novel coronavirus-infected pneumonia in Wuhan, China. JAMA 323, 1061-1069.

[9] Liu Y, Yang Y, Zhang C, Huang F, Wang F, Yuan J, Wang Z, Li J, Li J, Feng C, Zhang Z, Wang L, Peng L, Chen L, Qin Y, Zhao D, Tan S, Yin L, Xu J, Zhou C, Jiang C, Liu L (2020) Clinical and biochemical indexes from 2019-nCoV infected patients linked to viral loads and lung injury. Sci China Life Sci 63, 364-374.

[10] Xiong N, Wang T, Lin Z (2020) Invisible spread of SARS-CoV-2. Lancet Infect Dis. doi: 10.1016/S14733099(20)30263-2

[11] Isaia G, Marinello R, Tibaldi V, Tamone C, Bo M (2020) Atypical presentation of Covid-19 in an older adult with severe Alzheimer disease. Am J Geriatr Psychiatry 28, 790791.
[12] Wang H, Li T, Barbarino P, Gauthier S, Brodaty H, Molinuevo JL, Xie H, Sun Y, Yu E, Tang Y, Weidner W, Yu $X$ (2020) Dementia care during COVID-19. Lancet 395, 1190-1191.

[13] Hoffmann M, Kleine-Weber H, Schroeder S, Krüger N, Herrler T, Erichsen S, Schiergens TS, Herrler G, Wu NH, Nitsche A, Müller MA, Drosten C, Pöhlmann S (2020) SARS-CoV-2 cell entry depends on ACE2 and TMPRSS2 and is blocked by a clinically proven protease inhibitor. Cell 181, 271-280.e8.

[14] Kehoe PG, Wong S, Al MN, Palmer LE, Miners JS (2016) Angiotensin-converting enzyme 2 is reduced in Alzheimer's disease in association with increasing amyloid-beta and tau pathology. Alzheimers Res Ther $\mathbf{8}, 50$.

[15] Jiang T, Zhang YD, Zhou JS, Zhu XC, Tian YY, Zhao HD, Lu H, Gao Q, Tan L and Yu JT (2016) Angiotensin-(1-7) is reduced and inversely correlates with tau hyperphosphorylation in animal models of Alzheimer's disease. Mol Neurobiol 53, 2489-2497.

[16] Abdelkader NF, Abd EA, Khattab MM (2020) Telmisartan/17beta-estradiol mitigated cognitive deficit in an ovariectomized rat model of Alzheimer's disease: Modulation of ACE1/ACE2 and AT1/AT2 ratio. Life Sci 245, 117388 .

[17] Evans CE, Miners JS, Piva G, Willis CL, Heard DM, Kidd EJ, Good MA, Kehoe PG (2020) ACE2 activation protects against cognitive decline and reduces amyloid pathology in the Tg2576 mouse model of Alzheimer's disease. Acta Neuropathol 139, 485-502.

[18] Huq AJ, Fransquet P, Laws SM, Ryan J, Sebra R, Masters CL, Winship IM, James PA, Lacaze P (2019) Genetic resilience to Alzheimer's disease in APOE epsilon4 homozygotes: A systematic review. Alzheimers Dement 15, 1612-1623.

[19] Popovac A, Mladenović I, Krunić J, Trifković B, Todorović A, Milašin J, Despotović N, Stančić I (2020) Apolipoprotein $\varepsilon 4$ allele and dental occlusion deficiency as risk factors for Alzheimer's disease. J Alzheimers Dis 74, 797-802. 